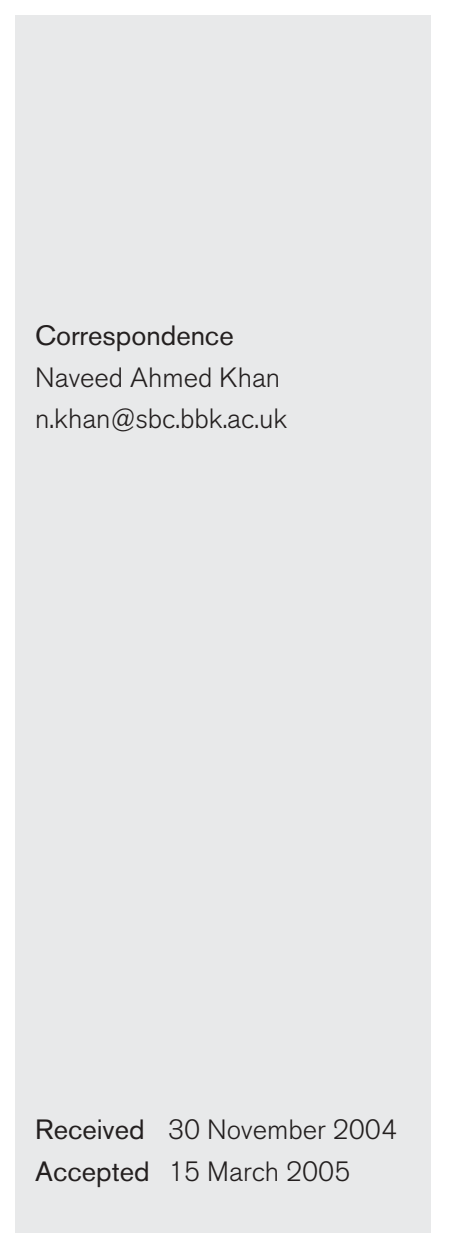

\title{
Acanthamoeba genotype T4 from the UK and Iran and isolation of the T2 genotype from clinical isolates
}

\author{
Amir Hossein Maghsood, ${ }^{1,2}$ James Sissons, ${ }^{2}$ Mostafa Rezaian, ${ }^{1}$ \\ Debbie Nolder, ${ }^{3}$ David Warhurst ${ }^{3}$ and Naveed Ahmed Khan ${ }^{2}$ \\ ${ }^{1}$ School of Biological and Chemical Sciences, Birkbeck College, University of London, \\ London WC1E 7HX, UK \\ ${ }^{2}$ Department of Medical Parasitology and Mycology, School of Public Health, Tehran University of \\ Medical Sciences, Tehran, 14155-6446, Iran \\ ${ }^{3}$ Diagnostic Parasitology Laboratory, London School of Hygiene and Tropical Medicine, London, UK
}

The majority of the keratitis-causing Acanthamoeba isolates are genotype T4. In an attempt to determine whether predominance of T4 isolates in Acanthamoeba keratitis is due to greater virulence or greater prevalence of this genotype, Acanthamoeba genotypes were determined for 13 keratitis isolates and 12 environmental isolates from Iran. Among 13 clinical isolates, eight (61.5\%) belonged to T4, two (15.3\%) belonged to T3 and three (23\%) belonged to the T2 genotype. In contrast, the majority of 12 environmental isolates tested in the present study belonged to T2 (7/12, $58.3 \%$ ), followed by $4 / 12$ T4 isolates (33.3\%). In addition, the genotypes of six new Acanthamoeba isolates from UK keratitis cases were determined. Of these, five (83.3\%) belonged to T4 and one was T3 (16.6\%), supporting the expected high frequency of T4 in Acanthamoeba keratitis. In total, the genotypes of 24 Acanthamoeba keratitis isolates from the UK and Iran were determined. Of these, 17 belonged to T4 (70.8\%), three belonged to T2 (12.5\%), three belonged to T3 (12.5\%) and one belonged to T11 (4.1\%), confirming that T4 is the predominant genotype $\left(\chi^{2}=4 \cdot 167\right.$; $P=0.0412)$ in Acanthamoeba keratitis.

\section{INTRODUCTION}

Acanthamoeba are ubiquitous organisms found in a variety of environments including soil, water, air, food items, humidifiers, dialysis units and healthy individuals (reviewed by Khan, 2003; Marciano-Cabral \& Cabral, 2003; Schuster \& Visvesvara, 2004). Based on their ecological distribution, there is circulation of Acanthamoeba strains between humans and the environment. Acanthamoeba are opportunistic causative agents of nasopharyngeal and cutaneous infections, painful blinding keratitis and fatal Acanthamoeba granulomatous encephalitis (Khan, 2003; Marciano-Cabral \& Cabral, 2003; Schuster \& Visvesvara, 2004). The difficulty in assigning an explicit role of Acanthamoeba in infections is because Acanthamoeba are heterogeneous and only certain subgroups may be pathogenic. Recent advances in the taxonomy of Acanthamoeba have led to the identification of the pathogenic subgroups. The genus Acanthamoeba has been classified into 15 different genotypes (T1-T15) based on rRNA gene sequencing (Gast, 2001; Stothard et al., 1998; Schuster \& Visvesvara, 2004). To date, only isolates belonging to the T3, T4, T6 and T11 genotypes have been associated

Abbreviation: HCEC, human corneal epithelial cell. with Acanthamoeba keratitis (Booton et al., 2002; De Jonckheere, 2003; Khan et al., 2002; Ledee et al., 1996; Stothard et al., 1998; Walochnik et al., 2000), and a large number of studies have identified $\mathrm{T} 4$ as the predominant keratitis-causing genotype. Indeed more than $90 \%$ of Acanthamoeba keratitis cases in literature reports have been caused by T4 isolates. As suggested before, the frequent involvement of T4 isolates in Acanthamoeba keratitis may be due to their greater abundance in the environment and thus they are most likely to be picked up by the susceptible hosts, or because $\mathrm{T} 4$ isolates possess properties that enable them to be more virulent, or both. In an attempt to clarify this, we determined the environmental and clinical distribution of Acanthamoeba in Iran. We tested 13 Acanthamoeba keratitis cases presented during 1998-2003 at the Tehran University of Medical Sciences, Iran. In addition, Acanthamoeba from environmental sources from widespread geographic locations were isolated and identified at the genotype level using rRNA gene sequencing.

\section{METHODS}

Clinical isolates. During 1998-2003, specimens from keratitis patients were tested for the presence of Acanthamoeba at the Department 
of Parasitology and Mycology, School of Public Health, Tehran University of Medical Sciences, Tehran, Iran. A total of 52 keratitis cases was tested. To determine the presence of Acanthamoeba, specimens (from contact lenses or corneal biopsies) were inoculated on to non-nutrient agar (Oxoid) plates seeded with Enterobacter aerogenes (formerly known as Klebsiella aerogenes). Plates were incubated at $30^{\circ} \mathrm{C}$ and observed daily for the presence of amoebae as previously described (Khan \& Paget, 2002).

Environmental isolates. Water samples from different cities in Iran (Table 1) were collected and tested for the presence of Acanthamoeba. For each sample, approximately $100-500 \mathrm{ml}$ water was filtered through a $0.45 \mu \mathrm{m}$ pore-size filter. Filters were inoculated on to non-nutrient agar plates overlaid with E. aerogenes as described above. Acanthamoeba were identified at the genus level, based on morphological characteristics of trophozoites and cysts using phase-contrast microscopy. Acanthamoeba were axenically cultivated in PYG medium $(0.75 \%$, $\mathrm{w} / \mathrm{v}$, proteose peptone; $0.75 \%, \mathrm{w} / \mathrm{v}$, yeast extract; and $1.5 \%$, w/v, glucose) at $30^{\circ} \mathrm{C}$ as described previously (Khan \& Paget, 2002).
Our previous studies have isolated Acanthamoeba from other geographic locations including the UK (Khan et al., 2002) and these isolates were compared in the present study. In addition, Acanthamoeba spp. were isolated from six new keratitis patients in the UK (Table 1), identified at the genotype level using rRNA gene sequencing and used in comparative studies.

PCR analysis. To confirm the identity of Acanthamoeba, PCR reactions were performed using genus-specific primers as previously described (Khan et al., 2001). Briefly, total DNA was extracted using the InstaGene matrix (Chelex; Bio-Rad) method as follows. Approximately $10^{3}$ cells (counted using a haemocytometer) were incubated with $30 \mu \mathrm{l}$ Chelex. Tubes were incubated at $56^{\circ} \mathrm{C}$ for $20 \mathrm{~min}$, followed by a $10 \mathrm{~min}$ incubation at $99.9^{\circ} \mathrm{C}$ and finally centrifuged at $10000 \mathrm{~g}$ for $5 \mathrm{~min}$. The supernatants containing DNA were used as the template for PCR. Primer sequences were 5'-TTTGAATTCGCTCCAATAGCGTATATT AA- $3^{\prime}$ and 5' -TTTGAATTCAGAAAGAGCTATCAATCTGT-3' (Kong \& Chung, 1996). PCR was performed in a volume of $50 \mu \mathrm{l}$ containing 1.25 U Taq polymerase (Qiagen), 0.1-1.0 ng DNA, $200 \mu \mathrm{M}$ dNTPs,

Table 1. Acanthamoeba isolates tested in the present study

\begin{tabular}{|c|c|c|c|c|}
\hline No. & Patient no. & Source & Species & Genotype \\
\hline \multicolumn{5}{|c|}{ (a) Environmental isolates (water samples) } \\
\hline 1 & $1-\mathrm{II}$ & Fountain pool water, Shariati square, Hamedan, Iran & Acanthamoeba palestinensis & $\mathrm{T} 2$ \\
\hline 2 & $3-\mathrm{I}$ & Fountain pool water, Imam square, Hamedan, Iran & A. palestinensis & $\mathrm{T} 2$ \\
\hline 3 & $6-\mathrm{V}$ & Pond water, Estakhr-e Abas-abad, Hamedan, Iran & Acanthamoeba castellanii & $\mathrm{T} 4$ \\
\hline 4 & $7-S$ & Pool water, Mellat Park, Hamedan, Iran & A. palestinensis & $\mathrm{T} 2$ \\
\hline 5 & 8 -II & Fountain pool water, Tehran, Tehran University, Iran & A. castellanii & $\mathrm{T} 4$ \\
\hline 6 & 8 -III & Fountain pool water, Tehran, Tehran University, Iran & $\mathrm{ND}^{*}$ & $\mathrm{ND}^{*}$ \\
\hline 7 & $9-S$ & Fountain pool water, Laleh Park, Tehran, Iran & A. palestinensis & $\mathrm{T} 2$ \\
\hline 8 & 16-I & Fountain pool water, Ramsar, Shahed University, Iran & A. palestinensis & $\mathrm{T} 2$ \\
\hline 9 & 19-I & Fountain pool water, Babataher square, Hamedan, Iran & A. palestinensis & $\mathrm{T} 2$ \\
\hline 10 & 21-II & Fountain pool water, Tonkabon, Imam square, Iran & A. castellanii & $\mathrm{T} 4$ \\
\hline 11 & 24-I & Fountain pool water, Ramsar, Shahrivar square, Iran & A. castellanii & $\mathrm{T} 4$ \\
\hline 12 & 27-II & Waterfalls, Ganj-nameh, Hamedan, Iran & A. palestinensis & $\mathrm{T} 2$ \\
\hline \multicolumn{5}{|c|}{ (b) Clinical isolates (keratitis patients) } \\
\hline 13 & 30 & Contact lens (Acanthamoeba keratitis patient), Iran & A. castellanii & $\mathrm{T} 4$ \\
\hline 14 & 52 & Contact lens (Acanthamoeba keratitis patient), Iran & A. castellanii & $\mathrm{T} 4$ \\
\hline 15 & 53 & Contact lens (Acanthamoeba keratitis patient), Iran & Acanthamoeba griffini & T3 \\
\hline 16 & 54 & Corneal tissue (Acanthamoeba keratitis patient), Iran & A. palestinensis & $\mathrm{T} 2$ \\
\hline 17 & 55 & Contact lens (Acanthamoeba keratitis patient), Iran & A. castellanii & $\mathrm{T} 4$ \\
\hline 18 & 56 & Contact lens (Acanthamoeba keratitis patient), Iran & A. griffini & T3 \\
\hline 19 & 58 & Contact lens (Acanthamoeba keratitis patient), Iran & A. castellanii & $\mathrm{T} 4$ \\
\hline 20 & 59 & Contact lens (Acanthamoeba keratitis patient), Iran & A. castellanii & $\mathrm{T} 4$ \\
\hline 21 & 60 & Contact lens (Acanthamoeba keratitis patient), Iran & A. castellanii & $\mathrm{T} 4$ \\
\hline 22 & 61 & Contact lens (Acanthamoeba keratitis patient), Iran & A. castellanii & $\mathrm{T} 4$ \\
\hline 23 & 62 & Contact lens (Acanthamoeba keratitis patient), Iran & A. castellanii & $\mathrm{T} 4$ \\
\hline 24 & 64 & Contact lens (corneal trauma by insect), Iran & A. palestinensis & $\mathrm{T} 2$ \\
\hline 25 & 65 & Contact lens (Acanthamoeba keratitis patient), Iran & A. palestinensis & $\mathrm{T} 2$ \\
\hline 26 & 515 & UK keratitis & A. griffini & T3 \\
\hline 27 & 771 & UK keratitis & A. castellanii & $\mathrm{T} 4$ \\
\hline 28 & 984 & UK keratitis & A. castellanii & $\mathrm{T} 4$ \\
\hline 29 & 1117 & UK keratitis & A. castellanii & $\mathrm{T} 4$ \\
\hline 30 & 1230 & UK keratitis & A. castellanii & $\mathrm{T} 4$ \\
\hline 31 & 1311 & UK keratitis & A. castellanii & $\mathrm{T} 4$ \\
\hline
\end{tabular}

*Acanthamoeba, but species/genotype not determined. This sample was omitted from the statistical analysis of association with genotype, i.e. only 11 environmental samples were included. 
$4 \mathrm{mM} \mathrm{MgCl}_{2}$ and $0.5 \mu \mathrm{M}$ primer. PCR reactions were performed at $94^{\circ} \mathrm{C}$ for $1 \mathrm{~min}, 55^{\circ} \mathrm{C}$ for $1 \mathrm{~min}$ and $72^{\circ} \mathrm{C}$ for $2 \mathrm{~min}$ for 30 cycles, with a final elongation step of $10 \mathrm{~min}$ at $72^{\circ} \mathrm{C}$. Amplified DNA was electrophoresed on a $2 \%$ agarose gel, stained and visualized under UV illumination.

185 rRNA gene sequencing. For $18 \mathrm{~S}$ rRNA gene sequencing, amplified DNA products were directly sequenced with an automated fluorescent sequencing system using a LI-COR 4200 DNA sequencer for diagnostic fragment 3 using primer 892C (5'-GTCAGAGGTGAA ATTCTTGG-3') (Booton et al., 2002) and identified at the genotype level by comparison with the available Acanthamoeba DNA sequences in GenBank or by comparison with the Acanthamoeba DNA sequence database (Department of Molecular Genetics, The Ohio State University, $\mathrm{OH}, \mathrm{USA}$ ). Diagnostic fragment 3 sequences for the new isolates are available upon request from N. A. Khan.

Cytotoxicity assay. For some Acanthamoeba isolates, cytotoxicity assays were performed as previously described (Khan \& Tareen, 2003). Briefly, immortalized human corneal epithelial cells (HCECs) were grown to confluency in 24-well plates in supplementary hormonal epithelial medium ( $15 \%$, w/v, fetal bovine serum; $40 \mu \mathrm{g}$ gentamicin $\mathrm{ml}^{-1} ; 5 \mu \mathrm{g}$ insulin $\mathrm{ml}^{-1} ; 0 \cdot 1 \mu \mathrm{g}$ cholera toxin $\mathrm{ml}^{-1}$; vitamins; $40 \%$ Hanks F12; 40 \% Dulbecco's modified Eagle's medium; 0.5 \% DMSO; $10 \mathrm{ng}$ epidermal growth factor $\mathrm{ml}^{-1}$ ) (Invitrogen) at $37^{\circ} \mathrm{C}$ in a $5 \% \mathrm{CO}_{2}$ incubator as previously described (Araki-Sasaki et al., 2000). Acanthamoeba isolates $\left(5 \times 10^{5}\right.$ amoebae per well) were incubated with cell monolayers in serum-free medium (RPMI 1640 containing $2 \mathrm{mM}$ glutamine, $1 \mathrm{mM}$ pyruvate and non-essential amino acids) at $37^{\circ} \mathrm{C}$ in a $5 \% \mathrm{CO}_{2}$ incubator for up to $24 \mathrm{~h}$. At the end of this incubation period, supernatants were collected and cytotoxicity was determined by measuring lactate dehydrogenase release using a cytotoxicity detection kit (Roche Applied Science) as previously described (Khan \& Tareen, 2003).

\section{RESULTS AND DISCUSSION}

\section{Isolation of Acanthamoeba from the clinical specimens in Iran}

Of 52 specimens taken from keratitis patients in Iran, 13 were found to contain Acanthamoeba, seven from women $(53.9 \%)$ and six from men $(46 \cdot 1 \%)$; their ages ranged from 15 to 54 years (mean 22 years). Twelve patients $(92.3 \%)$ wore soft contact lenses and one patient $(7 \cdot 7 \%)$ wore hard lenses. Patients were treated with topical application of propamidine isethionate (Brolene) plus Neosporin (neomycin, polymyxin B sulfates and bacitracin). Twelve patients responded to therapy with improved clinical symptoms and no report of recurrence, indicating successful treatment. One patient (\#64) did not respond to anti-amoebic drugs and a corneal graft was performed. This patient wore hard lenses and while travelling on a motorcycle an insect had entered his right eye. He rubbed his eye while wearing a contact lens, which resulted in corneal trauma. The clinical signs of Acanthamoeba keratitis followed soon after and included severe pain, photophobia and stromal infiltrates. Within 20 days, he was diagnosed with Acanthamoeba keratitis and anti-amoebic therapy was initiated but showed no response. However, the symptoms cleared after a successful corneal graft, as indicated above.
Isolation of Acanthamoeba from the environmental samples in Iran

To determine the distribution of Acanthamoeba in the environmental samples in Iran, 12 Acanthamoeba were isolated from water samples obtained from different cities (Table 1). Acanthamoeba were isolated based on morphological characteristics of trophozoites and cysts. Overall, these results suggested a wide environmental distribution of Acanthamoeba from various geographic locations in Iran. The identity of both clinical and environmental isolates was further confirmed using PCR analysis as described in Methods (data not shown). Acanthamoeba were successfully cultured in PYG medium containing penicillin $\left(100 \mathrm{U} \mathrm{ml}^{-1}\right)$ and streptomycin $\left(100 \mu \mathrm{g} \mathrm{ml}^{-1}\right)$ at $30^{\circ} \mathrm{C}$, except for one environmental isolate (8-III).

\section{Molecular typing of clinical isolates of Acanthamoeba from Iran}

In this study, 13 Acanthamoeba keratitis cases were identified. Among these, eight $(61.5 \%)$ isolates belonged to the T4 genotype, two $(15.3 \%)$ belonged to T3 and three $(23 \%)$ belonged to the T2 genotype (Table 2). This is the first time that Acanthamoeba isolates belonging to the T2 genotype have been linked with Acanthamoeba keratitis. Of interest, patient \#64, who was non-responsive to anti-amoebic therapy, was infected with a T2 isolate. To determine the pathogenic potential of the three T2 isolates (\#54, \#64 and $\# 65)$, in vitro cytotoxicity assays were performed. We observed that two Acanthamoeba isolates (\#64 and \#65) produced more than $90 \%$ HCEC cytotoxicity and were considered as potential pathogens. However, \#54 exhibited minimal HCEC cytotoxicity $(<10 \%)$ and was considered as a weak or non-pathogen. Overall, these data indicated that the majority of keratitis-causing isolates in Iran belong to the T4 genotype.

\section{T2 is a widely distributed genotype in the environmental samples isolated from Iran}

To determine the environmental distribution of Acanthamoeba in Iran, amoebae were isolated from 12 water samples. Out of these, seven $(58.3 \%)$ belonged to the T2 genotype and four belonged to T4 (33.3\%), while the Rns sequence of one (8-III) $(8 \cdot 3 \%)$ was not determined (Table 2). These findings indicated that $\mathrm{T} 2$ is a widely distributed genotype (followed by $\mathrm{T} 4)$ in the water samples tested from Iran.

\section{Clinical and environmental distribution of Acanthamoeba from other geographic locations}

For comparison of clinical isolates, 11 Acanthamoeba keratitis isolates from the UK were used (Khan et al., 2002, plus the six new Acanthamoeba keratitis cases tested in the present study; Table 1$)$. Of these, nine isolates $(81 \cdot 8 \%)$ belonged to the T4 genotype, one belonged to T3 $(9 \cdot 1 \%)$ and one belonged to T11 (9.1\%) (Table 2). In total, 24 Acanthamoeba keratitis isolates from the UK and Iran were tested. Of these 17 belonged to T4 (70.8 \%), three belonged to T2 $(12 \cdot 5 \%)$, 
Table 2. Comparison of the clinical and environmental distribution of Acanthamoeba in the UK and Iran

\begin{tabular}{|c|c|c|}
\hline Country & No. of samples & Genotype (\%) \\
\hline \multicolumn{3}{|c|}{ (a) Clinical isolates } \\
\hline \multirow[t]{3}{*}{ UK } & 11 & $1 \mathrm{~T} 3(9 \cdot 1)$ \\
\hline & & $9 \mathrm{~T} 4(81 \cdot 8)$ \\
\hline & & $1 \mathrm{~T} 11(9 \cdot 1)$ \\
\hline \multirow[t]{3}{*}{ Iran } & 13 & $3 \mathrm{~T} 2(23)$ \\
\hline & & $2 \mathrm{~T} 3(15 \cdot 3)$ \\
\hline & & $8 \mathrm{~T} 4(61 \cdot 5)$ \\
\hline \multirow[t]{4}{*}{ Total } & 24 & $174^{*}$ \\
\hline & & $3 \mathrm{~T} 2$ \\
\hline & & $3 \mathrm{~T} 3$ \\
\hline & & $1 \mathrm{~T} 11$ \\
\hline \multicolumn{3}{|c|}{ Association of $\mathrm{T} 4$ with clinical cases } \\
\hline $\mathrm{T}^{*}$ & Not T4 & Total \\
\hline 17 & 7 & 24 \\
\hline \multicolumn{3}{|c|}{ (b) Environmental isolates } \\
\hline \multirow[t]{4}{*}{ UK } & 9 & $2 \mathrm{~T} 2(22 \cdot 2)$ \\
\hline & & $1 \mathrm{~T} 3(11 \cdot 1)$ \\
\hline & & $4 \mathrm{~T} 4(44 \cdot 4)$ \\
\hline & & $2 \mathrm{~T} 7(22 \cdot 2)$ \\
\hline \multirow[t]{3}{*}{ Iran } & 12 & 7 T2 $(58 \cdot 3)$ \\
\hline & & $4 \mathrm{~T} 4(33 \cdot 3)$ \\
\hline & & $1 \mathrm{ND}$ \\
\hline \multirow[t]{5}{*}{ Total } & 21 & $8 \mathrm{~T} 4 \dagger$ \\
\hline & & $9 \mathrm{~T} 2$ \\
\hline & & $1 \mathrm{~T} 3$ \\
\hline & & $2 \mathrm{~T} 7$ \\
\hline & & Total: 20 \\
\hline \multicolumn{3}{|c|}{ Association of T4 with environment } \\
\hline $\mathrm{T} 4 \dagger$ & Not T4 & Total \\
\hline 8 & 12 & 20 \\
\hline
\end{tabular}

${ }^{*} \chi^{2}=4 \cdot 167$ with 1 degree of freedom. The two-tailed $P$ value equals $0 \cdot 0412$, indicating significant association of T4 with clinical cases.

$\dagger \chi^{2}=0 \cdot 800$ with 1 degree of freedom. The two-tailed $P$ value equals $0 \cdot 3711$, indicating no significant association of T4 in the environmental isolates.

three belonged to T3 $(12.5 \%)$ and one belonged to T11 $(4 \cdot 1 \%)$, confirming that T4 is the predominant genotype $\left(\chi^{2}=4 \cdot 167, P=0 \cdot 0412\right)$ in Acanthamoeba keratitis.

For comparison of the environmental isolates, nine Acanthamoeba isolates from the UK were tested as previously described (Khan et al., 2002; Khan \& Paget, 2002). Among these, four isolates $(44.4 \%)$ belonged to T4, two belonged to T2 $(22 \cdot 2 \%)$, one belonged to T3 $(11 \cdot 1 \%)$ and two belonged to T7 $(22 \cdot 2 \%)$ (Table 2). It is important to note that, except for the T4 isolates, the majority of amoebae were isolated from soil samples. Of interest, both T7 isolates were from soil samples.

Acanthamoeba are causative agents of painful eye infections, which can lead to blindness. They are one of the most ubiquitous organisms and have been classified into 15 different genotypes. In an attempt to correlate pathogenicity with certain genotypes, several studies have identified T4 as the major keratitis-causing genotype. However, whether the increased ability of T4 isolates to produce keratitis is due to their greater virulence or their greater prevalence is somewhat unclear. In this study, we tested clinical and environmental isolates of Acanthamoeba from a variety of sources and various geographic locations in the UK and Iran. The majority of clinical isolates tested in our study belonged to the T4 genotype, consistent with previous studies. Among the environmental isolates, T4 was again the predominant genotype in the UK, but T2 was predominant in Iran within the limited number of samples tested.

One of the interesting findings in our study was the isolation of T2 isolates from three keratitis patients (\#54, \#64, \#65). Of these, \#65 exhibited the characteristics of Acanthamoeba keratitis. In addition, \#65 received anti-acanthamoebic chemotherapy with a successful outcome, indicating Acanthamoeba as the causative agent. As quoted by De Jonckheere (2003) and others, 'contact lens cases are the breeding grounds for Acanthamoeba', and in the absence of a mixed infection, it is reasonable to presume that the T2 isolate was the causative agent. This is the first time that Acanthamoeba isolates belonging to the T2 genotype have been linked with Acanthamoeba keratitis. This is in contrast to previous studies, which showed that a T2 isolate (CCAP 1501/3c) exhibited minimal binding and minimal cytotoxicity to the host cells (Alsam et al., 2003; Khan et al., 2002) and was considered as a weak or non-pathogen. There are several possible explanations for these findings: the T2 genotype may consist of both pathogenic and weakly or non-pathogenic isolates (since rRNA gene sequence information is not a determinant for pathogenicity), or T2 isolates may have diverse virulence properties and individual strains (current or new isolates) should be tested for their virulence before designating them pathogens or non-pathogens. Alternatively, a more feasible scheme will be to subdivide the T2 genotype into two separate sequence types based on their Rns sequences. In support of this, Stothard et al. (1998) have shown that, within the $\mathrm{T} 2$ genotype, a sequence dissimilarity of $4.9 \%$ exists between CCAP $1501 / 3$ c and ATCC 30871. Comparison of CCAP $1501 / 3 \mathrm{c}$ with another T2 isolate (ATCC 50252) also revealed similar differences (4.5\%) (Stothard et al., 1998). These differences are very close to the current cut-off limit of $5 \%$ between different genotypes. Thus we propose that $\mathrm{T} 2$ isolates should be subdivided into two separate groups, T2A and T2B, with a sequence dissimilarity of $4 \%$ or more. This may help to differentiate pathogenic and non-pathogenic isolates within this genotype. Further studies involving Acanthamoeba belonging to the T2 genotype with CCAP 1501/3c-like Rns sequences and more detailed analysis of their properties will clarify the need for two separate groups in this genotype.

Another intriguing finding was the inability of one Acanthamoeba isolate (\#54 from the contact lens of a keratitis patient) to produce host-cell cytotoxicity, suggesting that it has weak 
and/or non-pathogenic properties $(<10 \%$ cell death, as compared with more than $70 \%$ cell death with other clinical isolates). One explanation for this is that there may have been a mixed infection and T2 was isolated from the contact lens even though it was not the causative agent. Future studies are in progress to address these issues.

Despite the occasional cases of Acanthamoeba keratitis due to T2 (the present study), T3 (Ledee et al., 1996), T6 (Walochnik et al., 2000) and T11 (Khan et al., 2002), the majority of cases are due to the T4 genotype. This was further confirmed in our study, as more than $80 \%$ of Acanthamoeba isolates (UK keratitis) belonged to the T4 genotype. In addition, we also determined that T4 was widely distributed in the environmental samples isolated from the UK. These results, together with previous findings, suggest that Acanthamoeba isolates belonging to the T4 genotype naturally occur in the environment and present potential reservoirs and therefore sources of infection to susceptible hosts. As indicated above, the repeated appearance of T4 isolates in keratitis may be due to their greater pathogenicity (virulence properties), greater transmissibility (i.e. they may be widely distributed in the habitat where the probability of being picked up by humans is very high), or a combination of both. To this end, we determined $\mathrm{T} 2$ as the most abundant genotype, followed by $\mathrm{T} 4$, in the environmental samples from Iran, even though the number of T4 Iranian keratitis isolates was higher than that of T2 isolates ( 61.5 vs $23 \%)$. These findings demonstrated that the abundance of T4 isolates in Acanthamoeba keratitis patients is most likely due to their greater virulence and/or properties that enhance their transmissibility, as well as their ability to bind to contact lenses and their decreased susceptibility to disinfectants. This raises additional questions about the properties of T2 and T4 genotypes that enable them to be the most widespread genotype in a given environment among 15 different genotypes and whether these properties play any role(s) in infection. Future studies that identify virulence traits and genetic markers limited to certain genotypes may help to clarify these issues.

Overall, there is a clear need for more detailed knowledge about the distribution of each genotype in different environments and for detailed analyses of direct and indirect virulence factors of environmental and clinical isolates of each genotype. These findings will help us understand the basis of differential distribution of genotypes in natural habitats, and to some extent in clinical cases, and may identify their differential properties contributing to disease, which should allow the development of pre-emptive or therapeutic measures against these serious infections.

\section{ACKNOWLEDGEMENTS}

We are grateful to Dr Sim Webb, Norwich Eye Research Group, School of Biological Sciences, University of East Anglia, UK, for providing immortalized human corneal epithelial cells and Dr Gregory Booton,
Department of Molecular Genetics, The Ohio State University, USA, for critical reviewing of the manuscript. This work was supported by grants from The Nuffield Foundation.

\section{REFERENCES}

Alsam, S., Kim, K. S., Stins, M., Rivas, A. O., Sissons, J. \& Khan, N. A. (2003). Acanthamoeba interactions with human brain microvascular endothelial cells. Microb Pathog 35, 235-241.

Araki-Sasaki, K., Aizawa, S., Hiramoto, M. \& 7 other authors (2000). Substance P-induced cadherin expression and its signal transduction in a cloned human corneal epithelial cell line. J Cell Physiol 182, 189-195.

Booton, G. C., Kelly, D. J., Chu, Y.-W., Seal, D. V., Houang, E., Lam, D. S. C., Byers, T. J. \& Fuerst, P. A. (2002). 18 S ribosomal DNA typing and tracking of Acanthamoeba species isolates from corneal scrape specimens, contact lenses, lens cases, and home water supplies of Acanthamoeba keratitis patients in Hong Kong. J Clin Microbiol 40, 1621-1625.

De Jonckheere, J. F. (2003). Epidemiological typing of Acanthamoeba strains isolated from keratitis cases in Belgium. Bull Soc Belge Ophtalmol 287, 27-33.

Gast, R. J. (2001). Development of an Acanthamoeba-specific reverse dot-blot and the discovery of a new ribotype. J Eukaryot Microbiol 48, 609-615.

Khan, N. A. (2003). Pathogenesis of Acanthamoeba infections. Microb Pathog 34, 277-285.

Khan, N. A. \& Paget, T. A. (2002). Molecular tools for speciation and epidemiological studies of Acanthamoeba. Curr Microbiol 44, 444-449.

Khan, N. A. \& Tareen, N. K. (2003). Genotypic, phenotypic, biochemical, physiological and pathogenicity-based categorisation of Acanthamoeba strains. Folia Parasitol 50, 97-104.

Khan, N. A., Jarroll, E. L. \& Paget, T. A. (2001). Acanthamoeba can be differentiated clinically by the polymerase chain reaction and simple plating assays. Curr Microbiol 43, 204-208.

Khan, N. A., Jarroll, E. L. \& Paget, T. A. (2002). Molecular and physiological differentiation between pathogenic and nonpathogenic Acanthamoeba. Curr Microbiol 45, 197-202.

Kong, H. H. \& Chung, D. I. (1996). PCR and RFLP variation of conserved region of small subunit ribosomal DNA among Acanthamoeba isolates assigned to either A. castellanii or A. polyphaga. Korean J Parasitol 34, 127-134.

Ledee, D. R., Hay, J., Byers, T. J., Seal, D. V. \& Kirkness, C. M. (1996). Acanthamoeba griffini. Molecular characterization of a new corneal pathogen. Invest Ophthalmol Vis Sci 37, 544-550.

Marciano-Cabral, F. \& Cabral, G. (2003). Acanthamoeba spp. as agents of disease in humans. Clin Microbiol Rev 16, 273-307.

Schuster, F. L. \& Visvesvara, G. S. (2004). Free-living amoebae as opportunistic and non-opportunistic pathogens of humans and animals. Int J Parasitol 34, 1001-1027.

Stothard, D. R., Schroeder-Diedrich, J. M., Awwad, M. H., Gast, R. J., Ledee, D. R., Rodriguez-Zaragoza, S., Dean, C. L., Fuerst, P. A. \& Byers, T. J. (1998). The evolutionary history of the genus Acanthamoeba and the identification of eight new $18 \mathrm{~S}$ rRNA gene sequence types. J Eukaryot Microbiol 45, 45-54.

Walochnik, J., Obwaller, A. \& Aspock, H. (2000). Correlations between morphological, molecular biological, and physiological characteristics in clinical and nonclinical isolates of Acanthamoeba spp. Appl Environ Microbiol 66, 4408-4413. 